\title{
Disturbance rejection via iterative learning control with a disturbance observer for active magnetic bearing systems
}

DOI:

10.1631/FITEE.1800558

\section{Document Version}

Accepted author manuscript

Link to publication record in Manchester Research Explorer

\section{Citation for published version (APA):}

Tang, Z. Z., Yu, Y. J., Li, Z. H., \& Ding, Z. T. (2019). Disturbance rejection via iterative learning control with a disturbance observer for active magnetic bearing systems. Frontiers of Information Technology \& Electronic Engineering, 20(1), 131-140. https://doi.org/10.1631/FITEE.1800558

\section{Published in:}

Frontiers of Information Technology \& Electronic Engineering

\section{Citing this paper}

Please note that where the full-text provided on Manchester Research Explorer is the Author Accepted Manuscript or Proof version this may differ from the final Published version. If citing, it is advised that you check and use the publisher's definitive version.

\section{General rights}

Copyright and moral rights for the publications made accessible in the Research Explorer are retained by the authors and/or other copyright owners and it is a condition of accessing publications that users recognise and abide by the legal requirements associated with these rights.

\section{Takedown policy}

If you believe that this document breaches copyright please refer to the University of Manchester's Takedown Procedures [http://man.ac.uk/04Y6Bo] or contact uml.scholarlycommunications@manchester.ac.uk providing relevant details, so we can investigate your claim.

\section{OPEN ACCESS}




\title{
Disturbance rejection via iterative learning control with a disturbance observer for active magnetic bearing systems
}

\author{
Ze-zhi TANG ${ }^{1}$, Yuan-jin YU ${ }^{1,2}$, Zhen-hong LI $^{1}$, Zheng-tao DING ${ }^{\dagger \ddagger 1,2}$ \\ ${ }^{1}$ School of Electrical and Electronic Engineering, University of Manchester, Manchester M13 9PL, United Kingdom \\ ${ }^{2}$ Sino-British Joint Advanced Control System Technology Laboratory, \\ James Lighthill Building, Manchester M13 9PL, United Kingdom \\ ${ }^{\dagger}$ E-mail: zhengtao.ding@manchester.ac.uk
}

Received Sept. 12, 2018; Revision accepted Dec. 25, 2018; Crosschecked Jan. 8, 2019

\begin{abstract}
Although standard iterative learning control (ILC) approaches can achieve perfect tracking for active magnetic bearing (AMB) systems under external disturbances, the disturbances are required to be iteration-invariant. In contrast to existing approaches, we address the tracking control problem of AMB systems under iteration-variant disturbances that are in different channels from the control inputs. A disturbance observer based ILC scheme is proposed that consists of a universal extended state observer (ESO) and a classical ILC law. Using only output feedback, the proposed control approach estimates and attenuates the disturbances in every iteration. The convergence of the closed-loop system is guaranteed by analyzing the contraction behavior of the tracking error. Simulation and comparison studies demonstrate the superior tracking performance of the proposed control approach.
\end{abstract}

Key words: Active magnetic bearings (AMBs); Iterative learning control (ILC); Disturbance observer https://doi.org/10.1631/FITEE.1800558

CLC number: TP27; TH133

\section{Introduction}

The maglev rotor system is collectively related to magnetic bearings (MBs), which make the rotor rotate without friction. MBs can be classified into active magnetic bearings (AMBs) and passive magnetic bearings (PMBs), of which AMBs provide some unique characteristics, including high rotation speed, low bearing losses, and lack of mechanical wear. Therefore, AMBs have attracted much attention in their broad applications such as vacuum pumps (Noh et al., 2005), spacecraft actuators (Sawada et al., 2001; Yu et al., 2015; Matsumura et al., 2016), and pumps that transport liquid in environments that have high cleanliness standards,

\footnotetext{
$\ddagger$ Corresponding author

(1) ORCID: Ze-zhi TANG, https://orcid.org/0000-0002-0182-6010 Zheng-tao DING, https://orcid.org/0000-0003-0690-7853

(C) Zhejiang University and Springer-Verlag GmbH Germany, part of Springer Nature 2019
}

like artificial hearts (Lee et al., 2003).

Disturbances exist widely in different AMB system processes, such as vibration of the external system, exogenous noises, and measurement uncertainties. These disturbances can severely affect the performance of the system, and even damage mechanical parts when a shaft rotates at an ultra-high speed. Thus, it is essential for AMBs to attenuate the effect of exogenous disturbances. There are many approaches that deal with the disturbance rejection of the AMB system. Matsumura et al. (1996) applied a loop-shaping design procedure to asymptotically reject disturbances caused by an unbalanced rotor. Lindlau and Knospe (2002) introduced an augmented linearized suspension plant and used a robust $\mu$ synthesis controller to counteract the independent disturbance force based on a feedback linearization model of voltage-controlled AMBs. Hong and Langari (2000) proposed a nonlinear magnetic bearing 
represented by a Takagi-Sugeno-Kang robust fuzzy model, in which harmonic disturbances and parameter uncertainties were considered.

However, for these modern robust control designs, the response of the system is not fast enough, and it is not easy for them to overcome severe plant uncertainties or disturbances because they cannot directly suppress system disturbances (Yang and Zheng, 2014). Meanwhile, disturbances of the actual AMB system exist periodically or have inherent characteristics, such as harmonics, which are not easily or promptly attenuated by traditional control designs. For these reasons, composite protocol combined modern control strategies with novel direct disturbance rejection tools, known as disturbance observers, have become more popular in recent years. These tools provide direct observation and rejection of system disturbances, and are considered a promising solution to address the intrinsic constraints of traditional feedback diagrams, such as nominal performance versus robustness (Chen et al., 2016). The superiority and convenience of disturbance observers have inspired researchers to explore their application in a magnetic bearings system. Yu et al. (2018b) proposed a disturbance observer based method that can attenuate the synchronous vibration of a magnetically suspended wheel. Yu et al. (2017) designed another instance of active vibration control of magnetically suspended wheels based on an active shaft deflection method. Peng et al. (2015) divided a dynamic AMB system into two equal parts, and used a robust controller and a disturbance observer to attenuate both matched and unmatched disturbances.

Most of the AMB system literature focuses on system stability issues, but does not deal with suspension tracking problems for suppression of the noise due to the relative motion of outer constructions. Some researchers discuss AMB system tracking problems. Yu et al. (2018a) discussed sliding mode based methods to deal with unmodeled matched coupling disturbances. Chladny and Koch (2008) proposed a nonlinear reduced-order disturbance observer incorporated into flatness-based tracking control. However, the AMB system motion is periodic and repetitive, so it is essential to consider repetitive-variant disturbances. Iterative learning control (ILC) is a type of system control that processes control tasks by repeatedly adjusting the control input. In each cycle, it processes the set algorithm from the error information collected in the previous or current cycle, thus leading the system offset to finally converge to zero. The controller acts like this to ensure that the system can follow specific trajectories. Broad applications have been implemented using the ILC algorithm. Bolder et al. (2012) successfully implemented the ILC algorithm in an inkjet printer. Baßler et al. (2015) described an application of a multi-body service robot combined with the ILC protocol. A dual-stage ILC system has been introduced to improve the performance of a multi-input multi-output (MIMO) unmatched system in joint elasticity robots, and there has been large-scale research on improving the ILC system. An overview of the development of the ILC system was available in Ahn et al. (2007). ILC is also widely applied in systems where similar tasks are repeated, such as motors (Mandra et al., 2015) and robotics (Zhao et al., 2015). It is also convenient to apply the ILC scheme in the AMB system to perform tracking goals cycle by cycle while rejecting exogenous disturbances.

Nevertheless, for the AMB system, the variation in the perturbation caused by shaft vibration would be hard for ILC to deal with alone. Because traditional ILC systems are focused on repetitive disturbances, there is no simple solution for a system with non-repetitive perturbation, such as random vibration in every period (Sun et al., 2014). On the other hand, for voltage-controlled AMBs, which are more widely used and affordable in real life, analysis shows that exogenous force disturbances usually affect the acceleration of the motion system, which is in a channel that is different from the control input. This is what we call the unmatched disturbance problem. The extended state observer (ESO) can actively estimate system disturbances without actual sensors, and requires only system input and output information. Thus, it is ideal for application in the AMB system. It is proved that ESO-based control can dynamically attenuate unmatched disturbances and improve the performance of systems that suffer from undesirable uncertainties. Therefore, in this study, an extended disturbance observer is introduced and combined with an ILC scheme to estimate and reject exogenous unmatched iteration variation disturbances of the AMB system. 


\section{Problem formulation}

Active magnetic bearings are usually built in five degrees of freedom (DOFs), with two radial translational DOFs, two radial torsional DOFs, and an axial translational DOF. The unbalanced factors, including vibration, external noises, and non-alignment between the mass center and the geometric center, could lead to runout of the rotor (Bi et al., 2005). Each of the translational DOFs can be an explicit single-degree-of-freedom (SDOF) model controlled by active electromagnets, while the control current operating the exogenous force is applied on the bearings. Thus, in this study, an unbalanced tracking problem for the radial SDOF model of AMBs is considered. Kucera (1997) proposed an SDOF nonlinear model of the AMBs (Fig. 1). Exogenous disturbances are in the form of external force $F_{s}$.

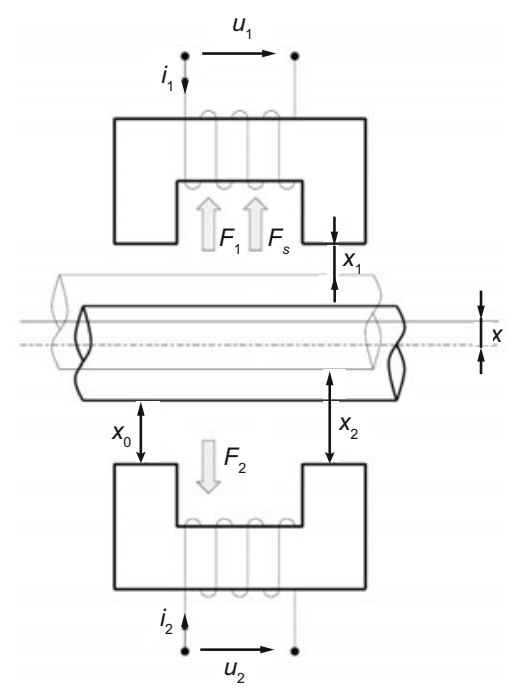

Fig. 1 Active magnetic bearing (AMB) model

In Fig. 1, rotor displacement along the $x$ axis is controlled by two electromagnetic poles working in differential mode. Control voltages $u_{1}$ and $u_{2}$ generate winding currents $i_{1}$ and $i_{2}$ of the upper pole and down pole, respectively, bringing magnetic forces $F_{1}$ and $F_{2}$, respectively. The external disturbance force is represented by $F_{s}$, while $x_{0}$ refers to the displacement when the rotor is in an equilibrium position. The magnetic gap lengths are represented by $x_{1}$ and $x_{2}$, while $x$ is the displacement deviation along the $x$ axis.

According to the Newton laws, the motion equation of the rotor can be derived as

$$
m \ddot{x}=F_{1}-F_{2}+F_{s},
$$

where $m$ is the mass of the rotor. According to the Ampere law, we have

$$
F_{j}=\frac{K}{4}\left(\frac{i_{j}}{x_{j}}\right)^{2}, j=1,2,
$$

where $K=\mu_{0} N^{2} A$, and $\mu_{0}, N$, and $A$ refer to the vacuum permeability, number of turns, and surface area, respectively.

As $x_{1}=x_{0}+x, x_{2}=x_{0}-x, i_{1}=i_{0}+i$, and $i_{2}=i_{0}-i$, we conclude that the electromagnetic force is

$$
\begin{aligned}
F_{1}-F_{2} & =\frac{K}{4}\left(\left(\frac{i_{1}}{x_{1}}\right)^{2}-\left(\frac{i_{2}}{x_{2}}\right)^{2}\right) \\
& =\frac{K}{4}\left(\left(\frac{i_{0}+i}{x_{0}+x}\right)^{2}-\left(\frac{i_{0}-i}{x_{0}-x}\right)^{2}\right) .
\end{aligned}
$$

Now, we linearize Eq. (3) at around $x=x_{0}$ and $i=i_{0}$ via the Taylor expansion, and can obtain

$$
F_{1}-F_{2}=\frac{\mu_{0} N^{2} A i_{0}}{x_{0}^{2}} i-\frac{\mu_{0} N^{2} A i_{0}^{2}}{x_{0}^{3}} x=k_{s} x+k_{i} i,
$$

where

$$
k_{s}=-\frac{K}{2} \frac{i_{0}^{2}}{x_{0}^{3}}, k_{i}=\frac{K}{2} \frac{i_{0}}{x_{0}^{2}} .
$$

The voltage drop of the system can be determined as

$$
u=R i+L_{s} \frac{\mathrm{d} i}{\mathrm{~d} t}+k_{i} \frac{\mathrm{d} x}{\mathrm{~d} t},
$$

where $R$ and $L_{s}$ are the referred resistance and inductance of the magnetic coils, respectively (Bleuler et al., 2009).

If we define velocity as $v=\dot{x}$, then the system states can be expressed as $\boldsymbol{x}=[x, v, i]^{\mathrm{T}}$. The thirdorder state equation we obtain can be described as

$$
\dot{\boldsymbol{x}}=\left[\begin{array}{ccc}
0 & 1 & 0 \\
\frac{2 k_{s}}{m} & 0 & \frac{2 k_{i}}{m} \\
0 & \frac{-k_{i}}{L_{s}} & \frac{-R}{L_{s}}
\end{array}\right] \boldsymbol{x}+\left[\begin{array}{c}
0 \\
0 \\
\frac{1}{L_{s}}
\end{array}\right] u+\left[\begin{array}{c}
0 \\
\frac{1}{m} \\
0
\end{array}\right] F_{s} .
$$

Putting it in the state-space form, we have

$$
\left\{\begin{aligned}
\dot{\boldsymbol{x}}_{k}(t) & =\boldsymbol{A} \boldsymbol{x}_{k}(t)+\boldsymbol{B}_{u} u_{k}(t)+\boldsymbol{B}_{d} d_{k}(t), \\
y_{k}(t) & =\boldsymbol{C} \boldsymbol{x}_{k}(t)
\end{aligned}\right.
$$

where $y_{k}(t), \boldsymbol{x}_{k}(t), u_{k}(t)$, and $d_{k}(t)$ represent the $k^{\text {th }}$ iteration displacement output, system state, 
control input, and external disturbances, respectively. Define $\boldsymbol{x}(t+k J)=\boldsymbol{x}_{k}(t), d(t+k J)=d_{k}(t)$, and $u(t+k J)=u_{k}(t)$, where $t \in[0, J], k \in \mathbb{N}$, and $J$ is a positive constant. Meanwhile, $\boldsymbol{A}, \boldsymbol{B}_{u}, \boldsymbol{B}_{d}$, and $\boldsymbol{C}$ represent the system matrix parameters; refer to Eq. (6), of which $\boldsymbol{C}=[1,0,0]$.

From the above state space form expression of the SDOF-AMB kinematic model, it can be found that all external constant factors, such as the mass of the rotor, are offset by part of the electromagnetic force, which comes from the bias current, or permanent magnetic force for some other traditional passive MBs. Therefore, control schemes need to be applied for adjustment of the electromagnet voltages, because it can easily be seen that the system is an unstable open loop. However, for most traditional control schemes like the proportional-integral-derivative (PID) controller, system runout would occur in certain initial conditions in a real system, caused by the non-alignment of the geometric center and mass center (Bi et al., 2005). Iterative learning schemes, referred to as ILC, can actively stabilize the system by refining the control input from the error information obtained by the previous cycle. Its repetitiveness can also compensate for the perturbation by tuning the control signal. For the AMB system, the designed ILC can generate a specific force to adjust the motion state of the bearings with the error information provided by the position sensors. The traditional ILC does not make direct use of disturbance information, but uses error information to compute the control input, which makes it difficult to deal with iteration-variant disturbances. Hence, it is essential to add the disturbance observer to observe and compensate for variant disturbances in every cycle; as for the real AMB system, perturbation mostly appears with random amplitude rather than a periodic signal.

\section{Mismatched disturbance estimation and rejection of an active magnetic bearing system}

\subsection{Common extended state observer design}

Disturbance observer based control (DOBC) has been widely proposed in the literature, and there is a detailed overview of diverse forms of disturbance observer design and related methods in Chen et al.
(2016). ESO is a kind of disturbance observer that can be designed with a minimum requirement of system information, which makes it widely applied and studied in various areas. For the proposed linear AMB system, only the order of the plant is required. A common ESO is formulated as follows.

Consider a system with order $n$ :

$$
\left\{\begin{aligned}
\dot{q}_{1} & =q_{2}, \\
\dot{q}_{2} & =q_{3} \\
& \vdots \\
\dot{q}_{n} & =a_{1} q_{1}+\ldots+a_{n} q_{n}+c \omega+b u, \\
p & =q_{1}
\end{aligned}\right.
$$

where $\omega$ and $u$ refer to disturbances and system input, respectively. It can be written as the state-space form:

$$
\left\{\begin{array}{l}
\dot{\boldsymbol{q}}=\overline{\boldsymbol{A}} \boldsymbol{q}+\overline{\boldsymbol{B}}_{u} u+\overline{\boldsymbol{B}}_{d} \omega \\
p=\overline{\boldsymbol{C}} \boldsymbol{q}
\end{array}\right.
$$

where $\boldsymbol{q}=\left[q_{1}, q_{2}, \ldots, q_{n}\right]^{\mathrm{T}}$,

$$
\begin{aligned}
& \overline{\boldsymbol{A}}=\left[\begin{array}{ccccc}
0 & 1 & 0 & \ldots & 0 \\
0 & 0 & 1 & \ldots & 0 \\
\vdots & \vdots & \vdots & & \vdots \\
a_{1} & a_{2} & a_{3} & \ldots & a_{n}
\end{array}\right], \\
& \overline{\boldsymbol{B}}_{u}=[0,0, \ldots, b]^{\mathrm{T}}, \\
& \overline{\boldsymbol{B}}_{d}=[0,0, \ldots, c]^{\mathrm{T}}, \\
& \overline{\boldsymbol{C}}=[1,0, \ldots, 0] \text {. }
\end{aligned}
$$

Then we augment the proposed equation by adding the state $q_{n+1}=a_{1} q_{1}+a_{2} q_{2}+\ldots+a_{n} q_{n}+c \omega$, and thus obtain $\dot{q}_{n}=q_{n+1}+b u, \quad \dot{q}_{n+1}=a_{1} \dot{q}_{1}$ $+\ldots+a_{n} \dot{q}_{n}+c \dot{\omega}$. An ESO can be designed as

$$
\dot{\boldsymbol{Z}}=\left[\dot{z}_{1}, \dot{z}_{2}, \ldots, \dot{z}_{n+1}\right]^{\mathrm{T}}=\boldsymbol{M} \boldsymbol{Z}+\boldsymbol{N} p+\boldsymbol{D} u
$$

where

$$
\boldsymbol{M}=\left[\begin{array}{ccccc}
-\alpha_{1} & 1 & 0 & \ldots & 0 \\
-\alpha_{2} & 0 & 1 & \ldots & 0 \\
\vdots & \vdots & \vdots & & \vdots \\
-\alpha_{n} & 0 & 0 & \ldots & 1 \\
-\alpha_{n+1} & 0 & 0 & \ldots & 0
\end{array}\right]
$$

$\boldsymbol{N}=\left[\alpha_{1}, \alpha_{2}, \ldots, \alpha_{n+1}\right]^{\mathrm{T}}, \boldsymbol{D}=[0, \ldots, b, 0]^{\mathrm{T}}$, where $z_{i}(i=1,2, \ldots, n)$ represent the estimates of the original system states, and $\alpha_{i}(i=1,2, \ldots, n)$ represent the observer gains that need to be designed. 
Thus, the estimation error can be calculated by subtracting the estimation value from the proposed system equations as

$$
\begin{aligned}
\dot{\boldsymbol{E}}= & {\left[\begin{array}{c}
\dot{e}_{1} \\
\dot{e}_{2} \\
\vdots \\
\dot{e}_{n+1}
\end{array}\right]=\left[\begin{array}{ccccc}
-\alpha_{1} & 1 & 0 & \ldots & 0 \\
-\alpha_{2} & 0 & 1 & \ldots & 0 \\
\vdots & \vdots & \vdots & & \vdots \\
-\alpha_{n} & 0 & 0 & \ldots & 1 \\
-\alpha_{n+1} & 0 & 0 & \ldots & 0
\end{array}\right] \boldsymbol{E} } \\
& +\left[\begin{array}{ccccc}
0 & 0 & \ldots & 0 & 0 \\
0 & 0 & \ldots & 0 & 0 \\
\vdots & \vdots & & \vdots & \vdots \\
-a_{1} & -a_{2} & \ldots & -a_{n} & 0
\end{array}\right] \dot{\boldsymbol{q}}_{n+1}+\left[\begin{array}{c}
0 \\
0 \\
\vdots \\
c
\end{array}\right] \dot{\omega}
\end{aligned}
$$

With a properly chosen observer gain, it was proved in Liu and $\mathrm{Li}$ (2012) that the stability is guaranteed if the system states, disturbances, and their derivatives are bounded.

\subsection{Universal extended state observer design}

However, for traditional ESO design, the proposed AMB model does not satisfy the standard form, because there is no integral framework. Meanwhile, for the AMB system, the exogenous disturbance force is applied in a different channel than the control input, which is widely known as the unmatched case. It is difficult to apply the standard DOBC form in a system with unmatched lumped disturbances.

Using system (7), a fundamental DOBC composite controller can be designed in the form of

$$
u_{k}^{*}(t)=u_{k}(t)-\frac{z_{n+1}}{b},
$$

where $u_{k}(t)$ is the controller designed to achieve system stability or for tracking purposes. Thus, it can obviously be observed that a direct compensation design cannot attenuate the influence of lumped disturbances from the output channel.

Remark 1 Although disturbances here can still be estimated, direct rejection is not applicable. Therefore, a generalized composite controller can be designed in the form $u_{k}^{*}(t)=u_{k}(t)+K_{d} \hat{\omega}$, and a new controller gain $K_{d}$ is calculated to attenuate the unmatched disturbances.

A generalized ESO can evolve from a framework similar to the basic ESO. If we define a new system state $x_{n+1}=d_{k}$ in system plant (7), an augmented plant can be acquired as

$$
\left\{\begin{aligned}
\dot{\tilde{\boldsymbol{x}}}_{k}(t) & =\tilde{\boldsymbol{A}} \tilde{\boldsymbol{x}}_{k}(t)+\tilde{\boldsymbol{B}}_{u} u_{k}(t)+\tilde{\boldsymbol{B}}_{d} \dot{d}_{k}(t), \\
y_{k}(t) & =\tilde{\boldsymbol{C}} \tilde{\boldsymbol{x}}_{k}(t)
\end{aligned}\right.
$$

where

$$
\begin{gathered}
\tilde{\boldsymbol{x}}_{k}(t)=\left[x_{1}, x_{2}, \ldots, x_{n+1}\right]^{\mathrm{T}}, \\
\tilde{\boldsymbol{A}}=\left[\begin{array}{cc}
\boldsymbol{A}_{n \times n} & \left(\boldsymbol{B}_{d}\right)_{n \times 1} \\
\mathbf{0}_{1 \times n} & \mathbf{0}_{1 \times 1}
\end{array}\right], \tilde{\boldsymbol{B}}_{u}=\left[\left(\boldsymbol{B}_{u}\right)_{1 \times n}, \mathbf{0}\right]^{\mathrm{T}}, \\
\tilde{\boldsymbol{B}}_{d}=\left[\mathbf{0}_{1 \times n}, \mathbf{1}\right]^{\mathrm{T}}, \tilde{\boldsymbol{C}}=\left[\boldsymbol{C}_{1 \times n}, \mathbf{0}\right] .
\end{gathered}
$$

In terms of realizing a generalized ESO design, the following assumptions are proposed:

Assumption 1 The disturbance $d_{k}$ and its derivation $\dot{d}_{k}$ are bounded, i.e., $\left\|d_{k}(t)\right\|_{2} \leq H_{1},\left\|\dot{d}_{k}(t)\right\|_{2} \leq$ $H_{2}$ for any $k \in \mathbb{N}$ over $t \in[0, \infty)$, where $H_{1}$ and $\mathrm{H}_{2}$ are positive constants, while the exogenous disturbance converges to a constant value during one period of the iterative cycle, i.e., $\lim _{t \rightarrow T} \dot{d}_{k}(t)=0$.

Assumption $2 \quad(\tilde{\boldsymbol{A}}, \tilde{\boldsymbol{C}})$ is observable.

A universal ESO can be designed as

$$
\left\{\begin{array}{l}
\dot{\hat{\tilde{\boldsymbol{x}}}}_{k}(t)=\tilde{\boldsymbol{A}} \hat{\tilde{\boldsymbol{x}}}_{k}(t)+\tilde{\boldsymbol{B}}_{u} u_{k}(t)+\boldsymbol{L}\left(y_{k}(t)-\hat{y}_{k}(t)\right), \\
\hat{y}_{k}(t)=\tilde{\boldsymbol{C}} \tilde{\boldsymbol{x}}_{k}(t),
\end{array}\right.
$$

where $\boldsymbol{L}$ is the observer gain to be designed. Comparing an augmented system with a generalized ESO, the estimation error can be obtained by the difference between two equations:

$$
\dot{\boldsymbol{E}}_{g}=(\tilde{\boldsymbol{A}}-\boldsymbol{L} \tilde{\boldsymbol{C}}) \boldsymbol{E}_{g}-\tilde{\boldsymbol{B}}_{u} \dot{d}_{k}(t)
$$

where $\boldsymbol{E}_{g}=\left[x_{1}, x_{2}, \ldots, x_{n}, d_{k}\right]^{\mathrm{T}}$ refers to the vector included estimation error information on the system state and exogenous disturbances.

Lemma 1 (Li et al., 2012) Supposing Assumptions 1 and 2 hold for Eq. (13), the error vector $\boldsymbol{E}_{g}$ is bounded if observer gain $\boldsymbol{L}$ is chosen to ensure that $(\tilde{\boldsymbol{A}}-\boldsymbol{L} \tilde{\boldsymbol{C}})$ is Hurwitz.

\section{Iterative learning control}

\subsection{Iterative learning control framework}

The basic idea for ILC is to create the control input of the present cycle by processing input and error information collected in the past and present cycles. In other words, it is a method that processes repetitive tasks based on the transient response of different sessions. Therefore, it is ideal to deal with repetitive work plant control problems. Recalling 
the state-space AMB system (7), we have

$$
\left\{\begin{aligned}
\dot{\boldsymbol{x}}_{k}(t) & =\boldsymbol{A} \boldsymbol{x}_{k}(t)+\boldsymbol{B}_{u} u_{k}(t)+\boldsymbol{B}_{d} d_{k}(t), \\
y_{k}(t) & =\boldsymbol{C} \boldsymbol{x}_{k}(t) .
\end{aligned}\right.
$$

Assumption $3 \boldsymbol{x}_{k}(0)=\mathbf{0}$, for any $k \in \mathbb{N}$.

Assumption $4 \quad\left(\boldsymbol{A}, \boldsymbol{B}_{u}\right)$ is controllable.

Suppose Assumptions 1-4 hold for Eq. (7) when $t \in[0, J]$, where $J$ is a positive constant (Sun and Li, 2017). The aim of the ILC system is to find an input, $u_{k}(t)$, to ensure that the trajectory $y_{k}$ converges to the real trajectory $y_{\mathrm{r}}$, when the cycles tend to infinity. The $k^{\text {th }}$ iteration tracking error $e_{k}(t)$ is defined by $e_{k}(t)=y_{\mathrm{r}}(t)-y_{k}(t)$.

Considering a classical ILC system like the following (Arimoto et al., 1984):

$$
u_{k}(t)=u_{k-1}(t)+P e_{k}(t)+Q e_{k-1}(t),
$$

where $P$ and $Q$ are the iterative learning gains that need to be designed, it can be simply derived that the initial status is $u_{0}(t)=0$.

\subsection{Tracking performance analysis}

To derive tracking errors, the Laplace transform is applied to Eq. (7):

$$
\left\{\begin{aligned}
s \boldsymbol{X}_{k}(s) & =\boldsymbol{A} \boldsymbol{X}_{k}(s)+\boldsymbol{B}_{u} U_{k}(s)+\boldsymbol{B}_{d} D_{k}(s) \\
Y_{k}(s) & =\boldsymbol{C} \boldsymbol{X}_{k}(s)
\end{aligned}\right.
$$

$$
\begin{aligned}
\boldsymbol{X}_{k}(s)= & (s \boldsymbol{I}-\boldsymbol{A})^{-1} \boldsymbol{B}_{u} U_{k}(s)+(s \boldsymbol{I}-\boldsymbol{A})^{-1} \boldsymbol{B}_{d} D_{k}(s), \\
Y_{k}(s)= & \boldsymbol{C} \boldsymbol{X}_{k}(s)=\boldsymbol{C}(s \boldsymbol{I}-\boldsymbol{A})^{-1} \boldsymbol{B}_{u} U_{k}(s) \\
& +\boldsymbol{C}(s \boldsymbol{I}-\boldsymbol{A})^{-1} \boldsymbol{B}_{d} U_{k}(s) \\
= & \Theta_{1}(s) U_{k}(s)+\Theta_{2}(s) D_{k}(s),
\end{aligned}
$$

where

$$
\left\{\begin{array}{l}
\Theta_{1}(s)=\boldsymbol{C}(s \boldsymbol{I}-\boldsymbol{A})^{-1} \boldsymbol{B}_{u} \\
\Theta_{2}(s)=\boldsymbol{C}(s \boldsymbol{I}-\boldsymbol{A})^{-1} \boldsymbol{B}_{d}
\end{array}\right.
$$

The tracking error can be obtained by calculating the difference between the reference trajectory and the control output of every cycle. Let $y_{\mathrm{r}}(t)$ be the reference signal, and $Y_{\mathrm{r}}(s)$ the Laplace transform of $y_{\mathrm{r}}(t)$. The tracking error can be represented as

$E_{k}(s)-E_{k-1}(s)=Y_{k}(s)-Y_{\mathrm{r}}(s)-\left(Y_{k-1}(s)-Y_{\mathrm{r}}(s)\right)$,

$$
\begin{aligned}
E_{k}(s)= & Y_{k}(s)-Y_{k-1}(s)+E_{k-1}(s) \\
= & E_{k-1}(s)-\left(\Theta_{1}(s) U_{k-1}(s)+\Theta_{2}(s) D_{k-1}(s)\right) \\
& +\left(\Theta_{1}(s) U_{k}(s)+\Theta_{2}(s) D_{k}(s)\right) \\
= & E_{k-1}(s)+\Theta_{1}(s)\left(U_{k}(s)-U_{k-1}(s)\right) \\
& +\Theta_{2}(s)\left(D_{k}(s)-D_{k-1}(s)\right) .
\end{aligned}
$$

According to Eq. (17), $U_{k}(s)-U_{k-1}(s)=P E_{k}(s)+$ $Q E_{k-1}(s)$ can be obtained. Hence, we can derive

$$
\begin{aligned}
E_{k}(s)= & \left(1+\Theta_{1}(s) P\right)^{-1} \Theta_{2}(s)\left(D_{k}(s)-D_{k-1}(s)\right) \\
& +\left(\left(1+\Theta_{1}(s) P\right)^{-1}\left(1-\Theta_{1}(s) Q\right)\right) E_{k-1}(s) .
\end{aligned}
$$

Theorem 1 For the designed ILC system, the tracking error converges asymptotically to a specific value if the learning parameters $P$ and $Q$ are set properly to ensure that $\left\|\left(1+\Theta_{1}(s) P\right)^{-1}\left(1-\Theta_{1}(s) Q\right)\right\|_{\infty} \leq 1$.

Proof From Eq. (24), we have

$$
\begin{aligned}
& \left\|e_{k}(t)\right\|_{2} \\
\leq & \left\|\left(1+\Theta_{1}(s) P\right)^{-1}\left(1-\Theta_{1}(s) Q\right)\right\|_{\infty}\left\|e_{k-1}(t)\right\|_{2} \\
& +\left\|\left(1+\Theta_{1}(s) P\right)^{-1} \Theta_{2}(s)\right\|\left\|_{\infty}\right\| d_{k}(t)-d_{k-1}(t) \|_{2} .
\end{aligned}
$$

It is clear that $\left\|\left(1+\Theta_{1}(s) P\right)^{-1}\left(1-\Theta_{1}(s) Q\right)\right\|_{\infty}$ and $\left\|\left(1+\Theta_{1}(s)\right)^{-1} \Theta_{2}(s)\right\|_{\infty}$ are bounded. Consider that the second term of inequality (25) is bounded due to Assumption 1, and thus the convergence of the ILC tracking error depends on the term $\left\|\left(1+\Theta_{1}(s) P\right)^{-1}\left(1-\Theta_{1}(s) Q\right)\right\|_{\infty}\left\|e_{k-1}(t)\right\|_{2}$. Assume that the second term of inequality (25) is bounded with

$$
\left\|\left(1+\Theta_{1}(s)\right)^{-1} \Theta_{2}(s)\right\|_{\infty}\left\|d_{k}(t)-d_{k-1}(t)\right\|_{2} \leq S_{1}
$$

There exists $R$ that satisfies

$$
\left\|\left(1+\Theta_{1}(s) P\right)^{-1}\left(1-\Theta_{1}(s) Q\right)\right\|_{\infty} \leq R<1 .
$$

In such a way, combined with inequality (26), we have

$$
\begin{aligned}
&\left\|e_{k}(t)\right\|_{2} \leq R\left\|e_{k-1}(t)\right\|_{2}+S_{1} \\
& \leq R^{2}\left\|e_{k-2}(t)\right\|_{2}+R S_{1}+S_{1} \\
& \leq R^{3}\left\|e_{k-3}(t)\right\|_{2}+R^{2} S_{1}+R S_{1}+S_{1} \\
& \vdots \\
& \leq R^{k}\left\|e_{0}\right\|_{2}+S_{1} \sum_{n=0}^{k-1} R^{n}
\end{aligned}
$$


From the first term of inequality $(27),\left\|e_{0}(t)\right\|_{2}$ is bounded, and

$$
\left\|\left(1+\Theta_{1}(s)\right)^{-1} \Theta_{2}(s)\right\|_{\infty}\left\|d_{k}(t)-d_{k-1}(t)\right\|_{2} \leq S_{1} .
$$

According to the sum algorithm of the geometric sequence, it can be concluded that the convergence of both terms is ensured if $R<1$.

Remark 2 If and only if $S_{1}=0$, namely, the exogenous disturbances are iteration-invariant, the learning gains $P$ and $Q$ can be chosen so that $\left\|\left(1+\Theta_{1}(s) P\right)^{-1}\left(1-\Theta_{1}(s) Q\right)\right\|_{\infty} \leq 1$, and the tracking error would converge to zero.

\section{Composite iterative learning control}

The tracking performance of the traditional ILC system was proved in Section 4. The universal ESO design in Section 3 provides a solution to attenuate disturbances in a different channel with control input.

Recall the generalized ESO composite controller. A similar form of the composite disturbance controller can be written as

$$
\bar{u}_{k}(t)=u_{k}(t)+T \hat{d}_{k}(t),
$$

where $T$ is the disturbance compensation gain that needs to be designed, and $\hat{d}_{k}(t)$ refers to the estimated iteration-variant disturbances.

If we replace new input Eq. (28) into the original AMB system (7), the Laplace transformed system tracking error can be displayed as

$$
\begin{aligned}
E_{k}(s)= & Y_{\mathrm{r}}(s)-Y_{k}(s)-\left(Y_{\mathrm{r}}(s)-Y_{k-1}(s)\right)+E_{k-1}(s) \\
= & Y_{k-1}(s)-Y_{k}(s)+E_{k-1}(s) \\
= & E_{k-1}(s)-\left(\Theta_{1}(s) \bar{U}_{k}+\Theta_{2}(s) D_{k}(s)\right) \\
& +\left(\Theta_{1}(s) \bar{U}_{k-1}(s)+\Theta_{2}(s) D_{k-1}(s)\right) \\
= & E_{k-1}(s)+\Theta_{1}(s)\left(\bar{U}_{k-1}(s)-\bar{U}_{k}(s)\right),
\end{aligned}
$$

and based on Eqs. (17) and (28), it can be derived that

$$
\begin{aligned}
E_{k}(s)= & \Theta_{2}(s)\left(D_{k-1}(s)-D_{k}(s)\right) \\
= & -\left(1+\Theta_{1}(s) P\right)^{-1} \Theta_{2}(s) \\
& \cdot\left(D_{k-1}(s)-D_{k}(s)-\left(\hat{D}_{k-1}(s)-\hat{D}_{k}(s)\right)\right) \\
& +\left(\left(1+\Theta_{1}(s) P\right)^{-1}\left(1-\Theta_{1}(s) Q\right)\right) E_{k-1}(s) \\
& +\left(\left(1+\Theta_{1}(s) P\right)^{-1}\left(T \Theta_{1}(s)+\Theta_{2}(s)\right)\right) \\
& \cdot\left(\hat{D}_{k-1}(s)-\hat{D}_{k}(s)\right) .
\end{aligned}
$$

Theorem 2 For the designed composed control system (28) with proper compensation gain $T$, the tracking error would be significantly reduced if the learning parameters $P$ and $Q$ are set to ensure that $\left\|\left(1+\Theta_{1}(s) P\right)^{-1}\left(1-\Theta_{1}(s) Q\right)\right\|_{\infty} \leq 1$.

Proof Likewise, based on the analysis in Section 4, we have

$$
\begin{aligned}
& \left\|e_{k}(t)\right\|_{2} \leq \\
& \left\|\left(1+\Theta_{1}(s) P\right)^{-1}\left(1-\Theta_{1}(s) Q\right)\right\|_{\infty}\left\|e_{k-1}(t)\right\|_{2} \\
& \left.+\| d_{k-1}(t)-d_{k}(t)-\hat{d}_{k-1}(t)+\hat{d}_{k}(t)\right) \|_{2} \\
& \cdot\left\|\left(1+\Theta_{1}(s)\right)^{-1} \Theta_{2}(s)\right\|_{\infty} \\
& +\left\|d_{k-1}(t)-d_{k}(t)\right\|_{2} \\
& \cdot\left\|\left(1+\Theta_{1}(s) P\right)^{-1}\left(T \Theta_{1}(s)+\Theta_{2}(s)\right)\right\|_{\infty} .
\end{aligned}
$$

It is obvious that the second and third terms are bounded when Assumptions 1 and 2 are satisfied. Assume

$$
\begin{aligned}
& \left.\| d_{k-1}(t)-d_{k}(t)-\hat{d}_{k-1}(t)+\hat{d}_{k}(t)\right) \|_{2} \\
& \cdot\left\|\left(1+\Theta_{1}(s)\right)^{-1} \Theta_{2}(s)\right\|_{\infty}+\left\|d_{k-1}(t)-d_{k}(t)\right\|_{2} \\
& \cdot\left\|I+\Theta_{1}(s) P^{-1}\left(T \Theta_{1}(s)+\Theta_{2}(s)\right)\right\|_{\infty} \leq S_{2} .
\end{aligned}
$$

It is proved in inequality (27) that the convergence of $e_{k}$ depends on the proper choice of learning gains $P$ and $Q$ to ensure $R<1$. The same results hold for inequality (32). The variable $R$ here refers to the maximum value of the term $\left\|\left(1+\Theta_{1}(s) P\right)^{-1}\left(1-\Theta_{1}(s) Q\right)\right\|_{\infty}$. Back to inequality (32), if the disturbance compensation gain is set as

$$
T=\left(\boldsymbol{C}(s \boldsymbol{I}-\boldsymbol{A})^{-1} \boldsymbol{B}_{u}\right)^{-1} \boldsymbol{C}(s \boldsymbol{I}-\boldsymbol{A})^{-1} \boldsymbol{B}_{d},
$$

together with inequality (32), we can obtain

$$
\begin{aligned}
& \left.\| d_{k-1}(t)-d_{k}(t)-\hat{d}_{k-1}(t)+\hat{d}_{k}(t)\right) \|_{2} \\
& \cdot\left\|\left(1+\Theta_{1}(s)\right)^{-1} \Theta_{2}(s)\right\|_{\infty} \\
= & \left\|\left(d_{k-1}(t)-\hat{d}_{k-1}(t)\right)-\left(\hat{d}_{k}(t)-d_{k}(t)\right)\right\|_{2} \\
& \cdot\left\|\left(1+\Theta_{1}(s)\right)^{-1} \Theta_{2}(s)\right\|_{\infty} \leq S_{2},
\end{aligned}
$$

and substituting it into inequality (31) gives

$$
\left\|e_{k}(t)\right\|_{2} \leq R^{k}\left\|e_{0}(t)\right\|_{2}+S_{2} \sum_{n=0}^{k-1} R^{n} \leq \frac{S_{2}-S_{2} R^{k-1}}{1-R} .
$$

Remark 3 Comparing inequalities (27) and (35), it can be seen that the tracking error of composite ILC is much smaller, because the estimation error of disturbances $\left(d_{k-1}(s)-\hat{d}_{k-1}(s)\right)-\left(\hat{d}_{k}(t)-d_{k}(t)\right)$ is surely smaller than $\left(d_{k-1}(s)-d_{k}(t)\right)$ if the ESO is properly designed. 


\section{Simulation results}

An AMB plant was built with the composite ILC system, based on the model that has been described. To prove the efficiency and superiority of the composite control scheme, iterative-variant-exogenous disturbances were introduced in the simulation, and the performance of each control scheme is shown and compared.

System parameters were set as: $k_{s}=9.5 \times 10^{5} \mathrm{~N} / \mathrm{m}, \quad k_{i}=675 \mathrm{~N} / \mathrm{A}, m=15 \mathrm{~kg}$, $L_{s}=0.025 \mathrm{H}, R=12 \Omega$, and thus the state-space model can be calculated.

The learning gain can be designed in PI form, which is $Q(s)=0.5+\frac{1}{s}, P=1.5+\frac{2.5}{s}$, and thus $\left\|\left(1+\Theta_{1}(s) P\right)^{-1}\left(1-\Theta_{1}(s) Q\right)\right\|_{\infty} \leq R<1$ is satisfied, and the disturbance compensation gain can be designed according to Eq. (33). The disturbance estimation gain is set as $\boldsymbol{L}=[-547.2,-340,-40,-60]^{\mathrm{T}}$. The iteration-variant disturbance force was set as

$$
d_{k}(t)=0.01 k+0.001 k \mathrm{e}^{(-2 t-1.4 k)} \sin (2 t-k),
$$

where $t \in[0,0.01]$ and $k \in \mathbb{N}$. Two sets of different reference trajectories were used in the simulation to prove the superiority and efficiency of the proposed control scheme.

The step response tracking performance of $r=$ $0.5 \mathrm{~mm}$ is shown in Figs. 2a-2d. The rotor position trajectories under different control schemes at four different iteration cycles $(k=2,5,10$, and 30) were demonstrated. It can be seen that the oscillation of the system trajectories gradually decreased for both systems, with the effect of the ILC algorithm. However, traditional ILC cannot offset the steady-state error, because the variability of the disturbances makes $\left\|d_{k-1}(t)-d_{k}(t)\right\|_{2}$ a variant value cycle by cycle. The composite control system, which combines ESO and ILC, quickly attenuated the disturbances, significantly reducing the steady-state error.

Figs. 3a-3d demonstrate the system tracking performance under the harmonics input reference

$$
r=0.5 \times 10^{-3}\left(1-\mathrm{e}^{-1200 t}\right) \sin \left(3 \times 10^{3} t\right),
$$

where $t \in[0,0.01], k \in \mathbb{N}$. It can be seen that the oscillation at the beginning of every cycle decreased as the number of cycles increased, and the system responses of two diverse control schemes show the same convergence rate. From both input signals, it
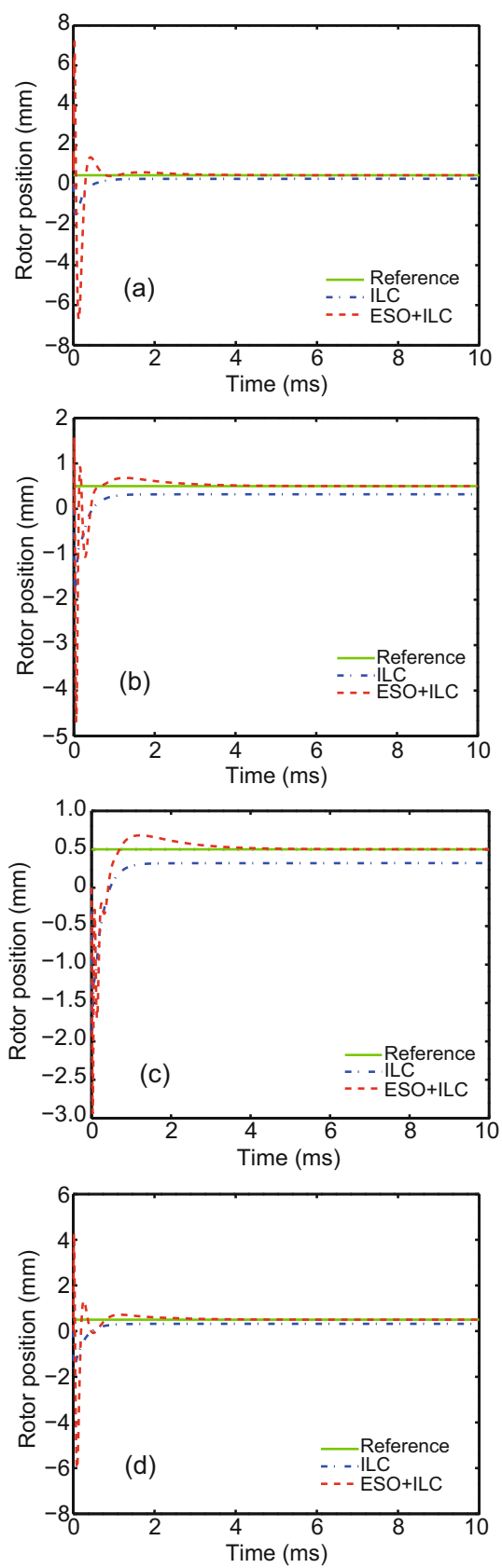

Fig. 2 Step tracking performance comparison for $k=2$ (a), $k=\mathbf{5}$ (b), $k=\mathbf{1 0}$ (c), and $k=\mathbf{3 0}$ (d) (ILC: iterative learning control; ESO: extended state observer)

can be seen that traditional ILC can roughly track the references with a steady-state error, while the observer-based composite control can reject exogenous disturbances to realize a more precise tracking goal. The influence of iteration-variant disturbance is proven to be eliminated. Compared to some existing results, the closed-loop-composite ILC can track set trajectories while rejecting exogenous disturbances. 

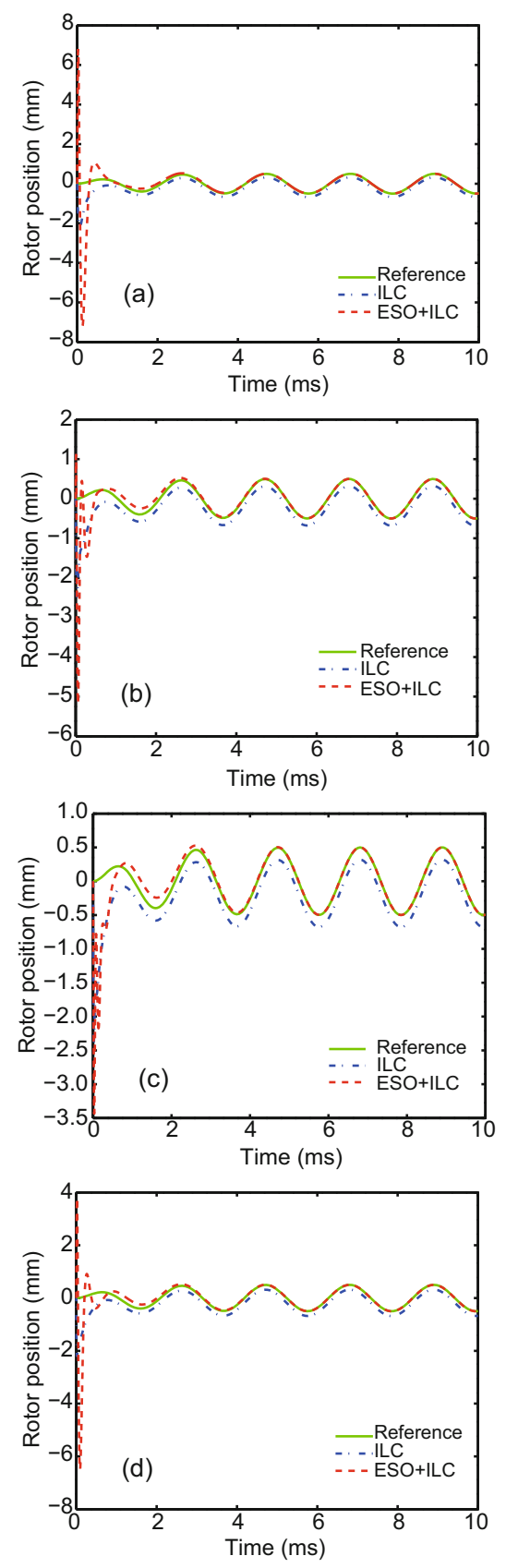

Fig. 3 Harmonics tracking performance comparison for $k=2$ (a), $k=5$ (b), $k=10$ (c), and $k=30$ (d) (ILC: iterative learning control: ESO, extended state observer)

\section{Conclusions}

In this study, a linearized model of voltage controlled AMBs has been analyzed under unmatched external disturbances. Then an integrated control scheme was proposed that combines a universal extended state observer and Arimoto-ILC and solves the unmatched problem improving the iteration-variant disturbance rejection ability, compared with the standard ILC system. Simulation results demonstrated that the proposed methods can achieve reference tracking while significantly attenuating the influence of unmatched-iteration-variant disturbances.

\section{References}

Ahn HS, Chen YQ, Moore KL, 2007. Iterative learning control: brief survey and categorization. IEEE Trans Syst Man Cybern Part C, 37(6):1099-1121. https://doi.org/10.1109/TSMCC.2007.905759

Arimoto S, Kawamura S, Miyazaki F, 1984. Bettering operation of robots by learning. J Field Robot, 1(2):123-140. https://doi.org/10.1002/rob.4620010203

Baßler S, Dünow P, Marquardt M, et al., 2015. Application of iterative learning control methods for a service robot with multi-body kinematics. $20^{\text {th }}$ Int Conf on Methods and Models in Automation and Robotics, p.465-470. https://doi.org/10.1109/MMAR.2015.7283920

Bi C, Wu DZ, Jiang Q, et al., 2005. Automatic learning control for unbalance compensation in active magnetic bearings. IEEE Trans Magn, 41(7):2270-2280. https://doi.org/10.1109/TMAG.2005.851866

Bleuler H, Cole M, Keogh P, et al., 2009. Magnetic Bearings: Theory, Design, and Application to Rotating Machinery. Springer-Verlag Berlin Heidelberg.

Bolder J, Lemmen B, Koekebakker S, et al., 2012. Iterative learning control with basis functions for media positioning in scanning inkjet printers. IEEE Int Symp on Intelligent Control, p.1255-1260. https://doi.org/10.1109/ISIC.2012.6398276

Chen WH, Yang J, Guo L, et al., 2016. Disturbance-observerbased control and related methods-an overview. IEEE Trans Ind Electron, 63(2):1083-1095. https://doi.org/10.1109/TIE.2015.2478397

Chladny RR, Koch CR, 2008. Flatness-based tracking of an electromechanical variable valve timing actuator with disturbance observer feedforward compensation. IEEE Trans Contr Syst Technol, 16(4):652-663. https://doi.org/10.1109/TCST.2007.912121

Hong SK, Langari R, 2000. Robust fuzzy control of a magnetic bearing system subject to harmonic disturbances. IEEE Trans Contr Syst Technol, 8(2):366-371. https://doi.org/10.1109/87.826808

Kucera L, 1997. Robustness of self-sensing magnetic bearing. Proc Industrial Conf and Exhibition on Magnetic Bearings, p.261-270.

Lee JH, Allaire PE, Tao G, et al., 2003. Experimental study of sliding mode control for a benchmark magnetic bearing system and artificial heart pump suspension. IEEE Trans Contr Syst Technol, 11(1):128-138. https://doi.org/10.1109/TCST.2002.806457

Li SH, Yang J, Chen WH, et al., 2012. Generalized extended state observer based control for systems with mismatched uncertainties. IEEE Trans Ind Electron, 59(12):4792-4802. https://doi.org/10.1109/TIE.2011.2182011

Lindlau JD, Knospe CR, 2002. Feedback linearization of an active magnetic bearing with voltage control. IEEE 
Trans Contr Syst Technol, 10(1):21-31.

https://doi.org/10.1109/87.974335

Liu HX, Li SH, 2012. Speed control for PMSM servo system using predictive functional control and extended state observer. IEEE Trans Ind Electron, 59(2):1171-1183. https://doi.org/10.1109/TIE.2011.2162217

Mandra S, Galkowski K, Aschemann H, et al., 2015. Guaranteed cost iterative learning control - an application to control of permanent magnet synchronous motors. IEEE $9^{\text {th }}$ Int Workshop on Multidimensional (nD) Systems, p.1-6. https://doi.org/10.1109/NDS.2015.7332639

Matsumura F, Namerikawa T, Hagiwara K, et al., 1996. Application of gain scheduled $H_{\infty}$ infinity robust controllers to a magnetic bearing. IEEE Trans Contr Syst Technol, 4(5):484-493. https://doi.org/10.1109/87.531915

Matsumura T, Kataza H, Utsunomiya S, et al., 2016. Design and performance of a prototype polarization modulator rotational system for use in space using a superconducting magnetic bearing. IEEE Trans Appl Supercond, 26(3):3602304. https://doi.org/10.1109/TASC.2016.2533584

Noh MD, Cho SR, Kyung JH, et al., 2005. Design and implementation of a fault-tolerant magnetic bearing system for turbo-molecular vacuum pump. IEEE/ASME Trans Mech, 10(6):626-631. https://doi.org/10.1109/TMECH.2005.859830

Peng C, Fang JC, Xu XB, 2015. Mismatched disturbance rejection control for voltage-controlled active magnetic bearing via state-space disturbance observer. IEEE Trans Power Electron, 30(5):2753-2762. https://doi.org/10.1109/TPEL.2014.2352366

Sawada H, Hashimoto T, Ninomiya K, 2001. High-stability attitude control of satellites by magnetic bearing wheels. Trans Jpn Soc Aeronaut Space Sci, 44(145):133-141. https://doi.org/10.2322/tjsass.44.133
Sun JK, Li SH, 2017. Disturbance observer based iterative learning control method for a class of systems subject to mismatched disturbances. Trans Inst Meas Contr, 39(11):1749-1760. https://doi.org/10.1177/0142331216645173

Sun JK, Li SH, Yang J, 2014. Iterative learning control with extended state observer for iteration-varying disturbance rejection. Proc $11^{\text {th }}$ World Congress on Intelligent Control and Automation, p.1148-1153. https://doi.org/10.1109/WCICA.2014.7052880

Yang J, Zheng WX, 2014. Offset-free nonlinear MPC for mismatched disturbance attenuation with application to a static var compensator. IEEE Trans Circ Syst II, 61(1):49-53. https://doi.org/10.1109/TCSII.2013.2290912

Yu YJ, Yang ZH, Fang JC, 2015. Medium-frequency disturbance attenuation for the spacecraft via virtual-gimbal tilting of the magnetically suspended reaction wheel. IET Contr Theory Appl, 9(7):1066-1074. https://doi.org/10.1049/iet-cta.2014.0578

Yu YJ, Yang ZH, Han C, et al., 2017. Active vibration control of magnetically suspended wheel using active shaft deflection. IEEE Trans Ind Electron, 64(8):65286537. https://doi.org/10.1109/TIE.2017.2682786

Yu YJ, Yang ZH, Han C, et al., 2018a. Fuzzy adaptive back-stepping sliding mode controller for high-precision deflection control of the magnetically suspended momentum wheel. IEEE Trans Ind Electron, 65(4):35303538. https://doi.org/10.1109/TIE.2017.2750617

Yu YJ, Yang ZH, Han C, et al., 2018b. Disturbance-observer based control for magnetically suspended wheel with synchronous noise. Contr Eng Pract, 72:83-89. https://doi.org/10.1016/j.conengprac.2017.10.019

Zhao YM, Lin Y, Xi FF, et al., 2015. Calibration-based iterative learning control for path tracking of industrial robots. IEEE Trans Ind Electron, 62(5):2921-2929. https://doi.org/10.1109/TIE.2014.2364800 